\title{
Capsule Commentary on Schickedanz et al., Impact of Social Needs Navigation on Utilization Among High-Utilizers in a Large Integrated Health System: a Quasi-Experimental Study
}

\author{
Seth A. Berkowitz, MD MPH \\ J Gen Intern Med 34(10):2582 \\ DOI: $10.1007 / \mathrm{s} 11606-019-05296-\mathrm{w}$ \\ (c) Society of General Internal Medicine 2019
}

Division of General Medicine \& Clinical Epidemiology, University of North Carolina School of Medicine, Chapel Hill, NC, USA.

T $\mathrm{n}$ this prospective evaluation of a large health-related social needs screening and navigation program for predicted high utilizers of healthcare services, Schickedanz et al. ${ }^{1}$ estimate a small decrease in healthcare utilization that is not statistically significant, when comparing those screened to all other predicted high utilizers. However, when restricting their analyses to subgroups with census tract indicators of lower socioeconomic status or with Medicaid insurance, they estimate both clinically meaningful and statistically significant reductions in healthcare utilization. The reasons for high utilization are quite heterogeneous, so examining subgroups more likely to have the health-related social needs that the intervention is meant to address makes sense. The results of this high-quality evaluation have important implications for both research and clinical operations within healthcare systems.

The interpretation of changes in healthcare utilization is complex. Healthcare is meant to increase life expectancy and/or improve quality of life. To the extent that decreased healthcare utilization represents preemption of problems that would otherwise have prompted healthcare use, then decreased utilization is good. But if decreased utilization represents lower use of beneficial services, then it may not be a good thing (losing one's health insurance may well decrease healthcare utilization, but I would not recommend it). In this case, since the intervention was unlikely to preclude access for necessary care, decreases in utilization likely represent improvements in participants' health.
All studies have limitations. In this case, because the intervention sites were not chosen at random, differences across sites could lead to bias. The authors attempted to account for this, but because individuals seen at the control sites could not receive the intervention, some bias is inherent to this study design. This bias may not be large enough to affect the findings, but it speaks to the need for further work in this area, ideally incorporating randomization.

In sum, the results presented here add to the mounting evidence that health-related social needs screening and navigation programs are important tools for improving health and healthcare. While, of course, more work remains to be done, Schickedanz et al. ${ }^{1}$ should be commended for a very valuable contribution to the field.

Corresponding Author: Seth A. Berkowitz, MD MPH; Division of General Medicine \& Clinical Epidemiology University of North Carolina School of Medicine, Chapel Hill, NC, USA (e-mail: seth_ berkowitz@med.unc.edu).

Compliance with Ethical Standards:

Conflict of Interest: The author declares that he does not have a conflict of interest.

\section{REFERENCE}

1. Schickedanz A, Sharp A, Hu YR, Shah NR, Adams JL, Francis D, Rogers A. Impact of Social Needs Navigation on Utilization Among HighUtilizers in a Large Integrated Health System: A Quasi-Experimental Study. J Gen Intern Med. 2019; https://doi.org/10.1007/s11606-01905123-2.

Publisher's Note Springer Nature remains neutral with regard to jurisdictional claims in published maps and institutional affiliations. 